https://helda.helsinki.fi

\title{
Long-term outcome of endoscopic shortening and stiffening of the vocal folds to raise the pitch
}

\section{Geneid, Ahmed}

$2015-12$

Geneid , A , Rihkanen , H \& Kinnari , T J 2015 , ' Long-term outcome of endoscopic shortening and stiffening of the vocal folds to raise the pitch ', European Archives of Oto-Rhino-Laryngology , vol. 272 , no. 12 , pp. 3751-3756 . https://doi.org/10.1007/s00405-015-3721-7

http://hdl.handle.net/10138/159085

https://doi.org/10.1007/s00405-015-3721-7

publishedVersion

Downloaded from Helda, University of Helsinki institutional repository.

This is an electronic reprint of the original article.

This reprint may differ from the original in pagination and typographic detail.

Please cite the original version. 


\title{
Long-term outcome of endoscopic shortening and stiffening of the vocal folds to raise the pitch
}

\author{
Ahmed Geneid $^{1} \cdot$ Heikki Rihkanen $^{1} \cdot$ Teemu J. Kinnari $^{1}$
}

Received: 3 April 2015/Accepted: 6 July 2015/Published online: 19 July 2015

(C) Springer-Verlag Berlin Heidelberg 2015

\begin{abstract}
In this study, we aimed to assess the long-term effects of the mucosal layer plastic surgery of the vocal folds performed on the voices of male-to-female transgenders. This retrospective cohort study enrolled 22 patients who were operated during 2004-2010 by a combined technique of transmucosal anterior suturing and stiffening of the vocal folds through a longitudinal cordotomy incision using $\mathrm{CO} 2$ laser. Long-term effects were assessed based on completed questionnaires on four different categories including subjective evaluation of patient's own voice perception and a standardized voice inventory (VHI), as well as an acoustic assessment and videolaryngoscopic examination. Out of the 22 patients contacted, 16 returned the questionnaires, and 13 of them participated in the subsequent acoustic analysis and videolaryngoscopic assessment. Results of the study were as follows: The total VHI score, after the procedure, was 32. F0 increased significantly for both the vowel/a/ and text from 157 to $207 \mathrm{~Hz}$ and 139 to $162 \mathrm{~Hz}$, respectively. Perturbation measures did not show a significant change. F0 did not correlate with the VHI score. One patient had symptomatic anterior perforation that needed re-operation. The combination of transmucosal anterior suturing and stiffening of the vocal folds by laser cordectomy results in significant elevation of the F0 in the long term and in acceptable levels of VHI
\end{abstract}

Electronic supplementary material The online version of this article (doi:10.1007/s00405-015-3721-7) contains supplementary material, which is available to authorized users.

Ahmed Geneid

ahmed.geneid@hus.fi

1 Department of Otorhinolaryngology and Phoniatrics, Head and Neck Surgery, Helsinki University Hospital and University of Helsinki, P.O. Box 220, 00029 Helsinki, Finland score compared to other reports. In conclusion, the results of the procedures show that it is enough to get the mucosal edges sutured together without the need to suture either the ligaments or the muscle of the vocal folds. The need for revision is minimal with this procedure.

Keywords Transgender - Transsexual · Glottoplasty · Pitch elevation · Voice feminization · Voice analysis

\section{Introduction}

A person with a desire to opt for higher voice pitch is a relatively recent challenge in phonosurgery. Most of these are male-to-female transgender people ( $\mathrm{M}$ to $\mathrm{F}$ ) who wish to have voice that is in harmony with the gender. Transsexualism is defined by World Health Organization as "desire to live and be accepted as a member of the opposite sex" [1]. One important tool for expression of gender and self-esteem is the voice [2]; according to their original sex, the male: female ratio of transsexuals is 3:1 in the western world [3]. A small minority of female-to-male transgenders need their voices' fundamental frequency lowered. This is done through the influence of androgen administration and is usually smooth and not problematic [4]. On the other hand, the vast majority of the $M$ to $F$ transgenders require a change in the pitch (fundamental frequency) and voice harmonics and to adopt the feminine type of communication skills. This is achieved either spontaneously or with the help of voice therapy. Only a small number are not satisfied with their voice or the fact that male voice still occurs in uncontrolled situations like yawning, laughing, coughing, and shouting. In addition, some cases have been reported of biologically born females needing pitch-raising surgery due to the low fundamental frequency of their voice. 
A number of surgical methods have been developed to raise the pitch. One of the most popular methods was developed by Isshiki in 1983 [5]. This method implies cricothyroid approximation. Thus, it results in increased tension of the vocal folds with a rise in the fundamental frequency. Another surgical approach to raise the fundamental frequency of voice was introduced by Gross in 1999 [6] based on previous works with similar ideas by Donald and Wendler [7, 8]. In this method, the anterior part of the vocal folds is sutured together. The suture is pulled through the muscle of the vocal folds lateral to the ligament. The idea is to shorten the functional length of the vocal folds. This results in changes of the voice box causing a rise in the fundamental frequency of the voice. A third technique was proposed by Orloff [9]. In this method, a lateral cordotomy incision is performed on the upper surface of the vocal folds using $\mathrm{CO} 2$ laser. This results in increased stiffness of the vocal folds. Thomas and McMillan have published the feminization laryngoplasty [10]. The aim is to diminish the vocal tract and enhance the feminine resonance. The anterior segments of the thyroid ala, vocal folds, and false cords are resected, a new anterior commissure created, and thyrohyoid approximation carried out.

A clear understanding of the best possible surgical method has been sought after. Despite the small number of operated $\mathrm{M}$ to $\mathrm{F}$ transgender people, no surgery is superior to the other, and the surgeon's focus is to confront a given problem: in this case to operating healthy vocal folds in order to create bettermatched voice. This surgery poses problems: the endolaryngeal sutures through the muscles are at risk of tearing through, the cricothyroid approximation is claimed to create an unnaturally tensed voice and diminished intonation with only temporal improvement. Thus, feminization laryngoplasty with laryngofissure requires further evaluation. Studies have reported good improvement on raising the fundamental frequency, however, this should be confirmed, and the risks of the operation analyzed before resection of the anterior part of the larynx can be suggested as a treatment of choice. The need for a surgical method that avoids the drawbacks of the previously mentioned ones motivated to develop the method presented in this study. This method has been practiced in Helsinki University Hospital for 10 years. The need to assess its longterm results motivated us to carry out this study.

\section{Aims}

The aim of this study was to assess the long-term effects of mucosal layer plastic surgery of the vocal folds on the voice of $\mathrm{M}$ to $\mathrm{F}$ transgenders, applying transmucosal anterior suturing to the vocal folds in combination with laser cordotomy incision on the mucosa and upper surface of the thyroarytenoid muscle.

\section{Subjects and methods}

\section{Surgery}

The idea of this surgery is to create a fusion of the anterior third of the vocal folds. The surgery is a modified combination of the techniques published by Gross and Orloff [6,9]. The most medial part of the mucosal edge of the anterior third of the vocal fold is removed creating a raw surface. Care should be given to leave the deeper part of the lamina propria. The remaining part of the mucosa, which is usually stronger than the removed most medial part, is then sutured together with two sutures without including the vocal ligament. We aim to remove and suture $50 \%$ of the vocal fold medial length. Based on our experience and findings, this is usually enough for fusion of the anterior part of the vocal folds combined with deep laser cordotomy. We have used 4-0 Vicryl with a straight needle which makes the endoscopic suturing considerably easier than sutures with a curved needle. This is followed by a longitudinal deep cordotomy incision using $\mathrm{CO} 2$ laser applying a technique similar to that introduced by Orlof but with a deeper incision extending to the level of the muscle. We feel comfortable using 3-5 $\mathrm{W}$ of a $\mathrm{CO} 2$ laser. The deep incision is thought to decrease the tension of the sutured mucosa allowing for its fusion primarily. Healing of the incision after that creates a scar on the upper side in order to increase the stiffness of the vocal fold as a secondary effect (The videos 1, 2, and 3 illustrate the steps of the procedure). The operation is carried out as day-care-surgery. During the recovery period of the first 2 weeks, the patient is advised to avoid unnecessary talking, physical stress, and fast deep breaths that may cause disruption of the sutured vocal fold mucosa. Resulting web and shape of the new glottis are shown here from two different patients (Figs. 1, 2).

\section{Subjects and methods of data collection}

The patient records of Helsinki University Hospital were reviewed retrospectively for the transgender patients who had undergone endolaryngeal shortening of the vocal folds during 2004-2010. A total of 22 subjects were identified and invited to participate in the study. All patients received voice therapy before the surgery, and were offered surgery when they found that results of voice therapy were insufficient to satisfy their demands. A questionnaire including the voice handicap index (VHI) [11] and questions on medical history and voice-related details were sent to all subjects.

\section{Questionnaires}

Questionnaires were completed by patients related to voice including questions on the ability to cope with different situations of communication, such as talking outdoors and 


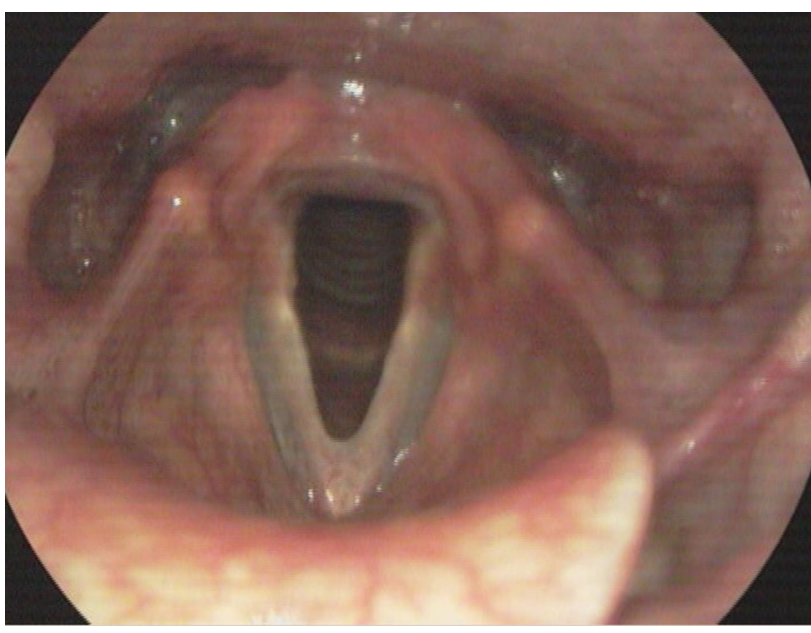

Fig. 1 Shape of the glottis of patient $(Y) 6$ years after the operations

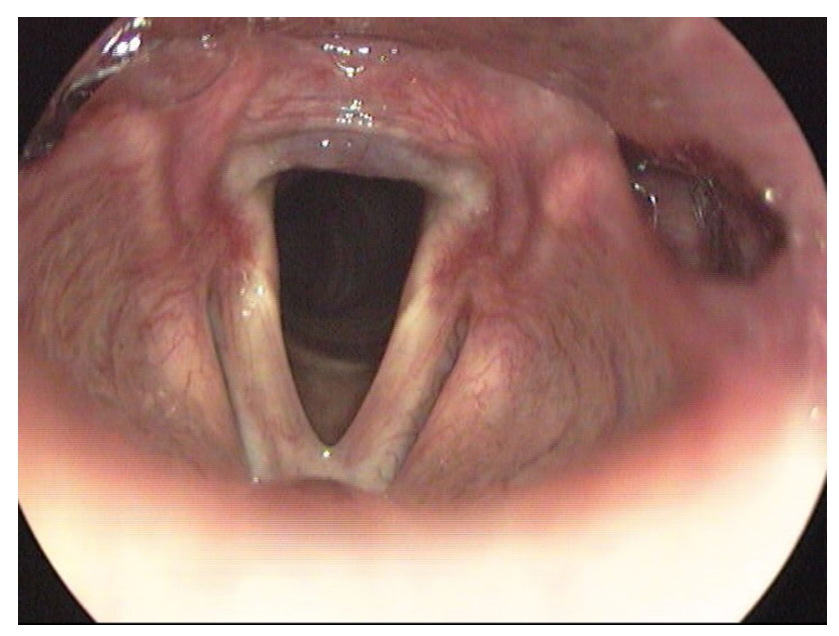

Fig. 2 Shape of the glottis of patient $(X) 2$ years after the operation

in noisy environments. Other queries included the assumption of gender when talking on the phone and the reaction of new people when meeting them and whether the patient's voice caused any confusion. A yes/no question on the patient's satisfaction with the current voice was included. Those who answered "no" were asked about the frequency with which their voice was considered masculine. In addition, an open question regarding the reason of dissatisfaction with their voice was included. The VHI was used to measure the psychosocial influence of voice.

\section{Acoustic analysis and videostroboscopy}

All subjects received an invitation for videostroboscopy. Videostroboscopy assessment included the following items: symmetry of the laryngeal structures at rest; symmetry of the vocal folds in movement; the presence of the anterior web created by the operation; the assessment of mucosal wave in terms of symmetry, phase, and closure.

Prior to the operation, voice recording was done for most of the subjects, and it was carried out for all subjects who participated in the laryngeal examination for this assessment. Both pre and postoperative recordings were performed in a soundproof booth. Mouth to microphone distance was $30 \mathrm{~cm}$. The setup of a Casio Digital Audio Tape (DAT) recorder was kept constant during recordings. Recordings were transferred to a laptop using a portable hard disk player (iRiver 140, Iriver Ltd., Irvine, CA, USA). Acoustic analysis of the voice recordings was done using Praat [12]. Sustained vowel/a/, at most comfortable pitch and loudness level, as well as a text of 43 words were analyzed for F0. Vowel/a/was analyzed also for Jitter (i.e., cycle-to-cycle frequency perturbation), Shimmer (i.e., cycle-to-cycle amplitude perturbation), and NHR (an expression of aperiodic-to-periodic sound) [13].

The study received the approval of the ethics committee of the Helsinki University Hospitals.

Statistical analysis was performed using Statistical Program for Social Sciences (SPSS for Windows, Version 20.0 statistical software; SPSS Inc, Chicago, IL, USA). Paired $t$ test analysis was used to compare the changes in the acoustic measurements of the voice recordings. Pearson correlation was used to test correlation between age, VHI, acoustic measurements, and lengths of the vocal folds.

\section{Results}

A total of 16 (out of 22) subjects returned completed questionnaires and VHIs. Only two subjects reported to be smokers. Five subjects $(31 \%)$ expressed their complete satisfaction with the results of the operation. The rest complained that their voice is often masculine in 6 cases, sometimes in 3 cases, rarely in one case, and never in one case. The remaining one, who was not satisfied with her voice although not sounding masculine, expressed that the reason for dissatisfaction was the inability to raise her voice and shout.

When asked how often they were considered women on the phone. Nine (56\%) subjects said often, while 7 (44\%) reported on less frequent occasions. Among these 7 choices were sometimes (1), rarely (1), never (1), or was not able to say (4).

Four $(25 \%)$ subjects reported that voice often causes confusion when meeting new people; 4 (25\%) reported sometimes; and $5(31 \%)$ rarely. Three $(19 \%)$ subjects thought that this never happens. For several subjects, the ability to use voice in noisy environments and outdoors was diminished as shown in Table 1. 
Table 1 Percentage of subjects suffering from reduced ability of the voice to cope with different situations of communication

\begin{tabular}{llllll}
\hline $\begin{array}{l}\text { Type of situation and its effect on } \\
\text { voice }\end{array}$ & $\begin{array}{l}\text { Doesn't disturb } \\
(\%)\end{array}$ & $\begin{array}{l}\text { Disturb slightly } \\
(\%)\end{array}$ & $\begin{array}{l}\text { Disturb moderately } \\
(\%)\end{array}$ & $\begin{array}{l}\text { Disturb remarkably } \\
(\%)\end{array}$ & $\begin{array}{l}\text { Prevent talking } \\
(\%)\end{array}$ \\
\hline Talking in noisy places & 19 & 19 & 25 & 31 & 6 \\
Talking outdoors & 44 & 31 & 6 & 19 & 0 \\
Talking for a long time & 75 & 0 & 0 & 19 & 6 \\
\hline
\end{tabular}

\section{VHI}

The mean scores of the functional, physical, and emotional parts of the VHI were $10,11,10$, respectively. The mean of the total score was 32 .

\section{Acoustic analysis and videostroboscopy}

Of the 16 subjects, 13 participated in the laryngeal examination and voice recordings. Eight of them had their preoperative voice recordings available. Accordingly, the acoustic analysis was limited to those patients whose voices have been recorded both before the operation and postoperatively for this study. The F0 obtained from the acoustic analysis of the text read showed that average of F0 increased from 130 to $162 \mathrm{~Hz}$. The mean F0 obtained from vowel/a/increased from 157 to $207 \mathrm{~Hz}$. Jitter decreased from 0.427 to 0.367 . Also Shimmer decreased from 9.919 to 5.327. NHR decreased from 0.118 to 0.026 . Changes observed in the acoustic analysis were not significant except for the F0 of the text sample $(p=0.009)$ and the vowel/a/ $(p=0.025)$. By adding the five patients who participated only postoperatively in the voice recording, the whole group of 13 patients had a mean F0 obtained from the text of $169 \mathrm{~Hz}$ and from the vowel/a/206 Hz (Fig. 3).

Twelve subjects were examined by videostroboscopy using a rigid scope. One had an intense gag reflex due to which the examination was performed using fiber optics without stroboscopy. Laryngeal and vocal fold vibration and movement symmetry was confirmed in all subjects. All the 12 subjects who underwent stroboscopy demonstrated symmetric vocal fold mucosal wave. Four subjects showed complete vocal fold closure; the rest had different shapes of gap between the vocal folds. The most common was an anterior gap (5 subjects). Two had an oval-shaped glottis opening, and one incomplete closure anteriorly and from the middle. One subject had an hour-glass pattern of the glottis during phonation with the glottis open anteriorly and posteriorly.

Out of the 13 subjects examined, 1 had a symptomatic anterior perforation in addition to granulation tissue in its edge, which was re-operated. There were 4 other patients

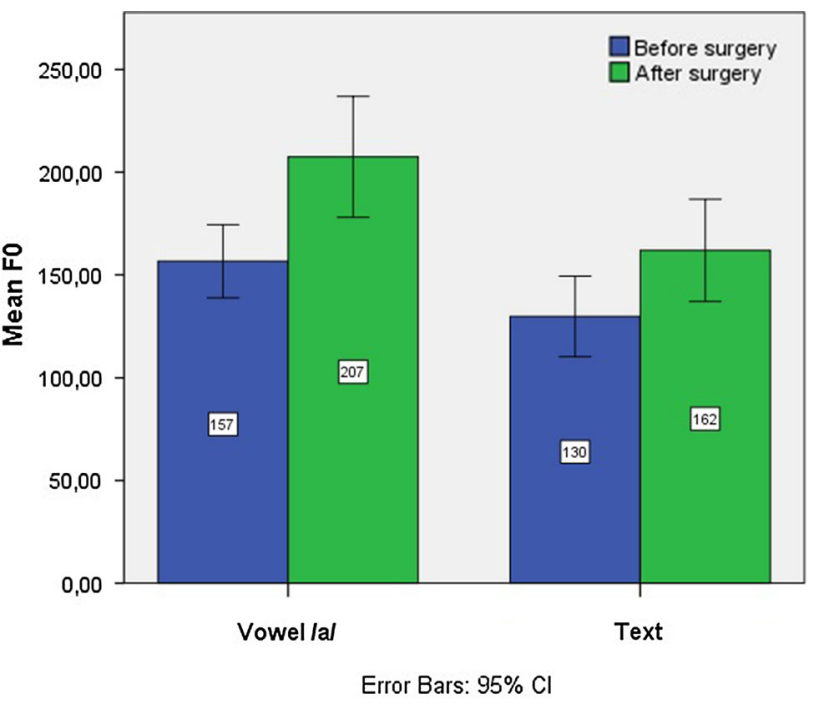

Fig. 3 Mean F0 before and after surgery for vowel/a/ and text

who presumably had a small superficial perforation or notch on the upper surface of the web; however, neither associated impact on voice nor symptoms were seen.

The VHI correlation to the measured F0 was tested and found to be insignificant. We also tested the correlation of age to VHI and F0 change, and none was found to be significant.

\section{Discussion}

In our hospital, all $\mathrm{M}$ to $\mathrm{F}$ transgenders are primarily invited for voice therapy. Only those who are not satisfied with the results of voice therapy are possible candidates for glottoplasty. This study was a retrospective examination of the long-term effects of a combined endolaryngeal shortening and scarring of the vocal folds offered to those $\mathrm{M}$ to $\mathrm{F}$ transgenders who went through gottoplasty. Four different assessment categories were utilized, including subjective evaluation of patient's own perception of how they sound; a standardized voice inventory (VHI); an acoustic assessment; and videolaryngoscopic examination. 
After cricothyroid approximation, the level of satisfaction ranges from 55 to $78 \%[14,15]$. Systematic evaluation of the satisfaction of $\mathrm{M}$ to $\mathrm{F}$ transgenders who underwent Wendeler's technique, or its modification has been usually done by applying the VHI questionnaire [7, 8] We observed a mean VHI total score after procedure of 32 , which is better than that found in previous studies, but remaining far from normal. Remacle et al. [16] found that VHI scores prior to and post surgery were 45.5 , and 42 , respectively. Another study $(N=31)$ also documented quite similar, poor scores before surgery (47) and even worse (54) after it [17]. Orloff et al. [9] examined the VHI scores postoperatively $(N=13)$ having used a method to stiffen the vocal folds by $\mathrm{CO} 2$ laser scarring; they observed a mean VHI score of 51. VHI was not measured in the study by Thomas and MacMillan [10]. In the present study, the mean VHI total score after the procedure was 32 . The figure is better than that found in the previous studies.

We also examined patient satisfaction through a specially tailored questionnaire. We found that $31 \%$ were completely satisfied with the results of the operation, $56 \%$ had the feeling that they were often considered as women on the phone, and $75 \%$ thought that their voice did not often cause confusion when meeting with new people.

Acoustic analysis showed that F0 increased significantly for both the read-text sample as well as the sustained vowel/a/. The average F0 of the text prior to the procedure was $130 \mathrm{~Hz}$ and rose to $162 \mathrm{~Hz}$. This is not as marked as in sustained vowel/a/ in the reported series. In the present study, the difference between the text and vowel/a/ in terms of the F0 can be attributed to the subjects' ability, and possibly to their desire to maintain a higher pitch on sustained vowels.

The F0 of vowel/a/ $(207 \mathrm{~Hz})$ after surgery comes in accordance with previous studies and expectations in which the level of F0 after surgery was $190-240 \mathrm{~Hz}$ [1618]. F0 of text after surgery was lower than expected $(162 \mathrm{~Hz})$ compared to the results of feminization laryngoplasty by Thomas and MacMillan $(196 \mathrm{~Hz})$ [10]. This may be not only because the procedure was not aggressive enough, but also because Finnish culture favors low pitches among speakers [19] and speakers acknowledge this by trying to keep their voice in the low female level. It is also worth mentioning that female Finnish speakers tend to have lower speaking F0 [20] in comparison with American peers [21].

One of the 13 subjects who were operated on had an anterior perforation and had to undergo a revision surgery. This percentage of revision $(7 \%)$ is similar to that reported from the Wendler technique (10\%) [17].

Of particular interest is that despite our expectation to find correlation between age and F0 change or between F0 and VHI, none was found to be significant. This sheds light on the fact that an increase in the F0 does not mean a better VHI in this group of patients. It also goes against our clinical impression that a young patient may end up having a better F0.

\section{Conclusion}

The rarity of studies that examine long-term effects of shortening of the vocal folds among $\mathrm{M}$ to $\mathrm{F}$ transgenders limits the ability to compare the results of the surgical intervention. However, the results of this study show good endoscopic outcomes with significant increase of the F0 on both text and vowel/a/ recordings. The results also show that it is enough to have the mucosa of the anterior thirds intact without the need to have the sutures going through the muscle or ligament of the vocal fold. The degree of satisfaction measured by the voice handicap index (VHI) inventory is better than that of other studies carried on other types of surgical procedures. Nevertheless, the need for re-operation/revision is quite limited.

Acknowledgments We would like to extend our gratitude to the Finnish Ear, Nose and Throat Research Foundation for their financial support to this study.

\section{Compliance with ethical standards}

Conflict of interest The authors have no financial relationships, or conflicts of interest to disclose.

\section{References}

1. World Health Organisation (2015) International statistical classification of diseases and related health problems, version 2015. WHO, Geneva

2. Hancock AB, Krissinger J, Owen K (2011) Voice perceptions and quality of life of transgender people. J Voice 25:553-558

3. Van Kesteren PJ, Gooren LJ, Megens JA (1996) An epidemiological and demographic study of transsexuals in the Netherlands. Arch Sex Behav 25:589-600

4. Van Borsel J, de Pot K, De Cuypere G (2009) Voice and physical appearance in female-to-male transsexuals. J Voice 23:494-497

5. Isshiki N, Taira T, Tanabe M (1983) Surgical alteration of the vocal pitch. J Otolaryngol 12:335-340

6. Gross M (1999) Pitch-raising surgery in male-to-female transsexuals. J Voice 13:246-250

7. Donald PJ (1982) Voice change surgery in the transsexual. Head Neck Surg 4:433-437

8. Wendler J (1989) Vocal pitch elevation after transsexualism male to female. In: XVI Union of the European Phoniatricians Congress, Salsomaggiore, Italy

9. Orloff LA, Mann AP, Damrose JF, Goldman SN (2006) Laserassisted voice adjustment (LAVA) in transsexuals. Laryngoscope 116:655-660

10. Thomas JP, Macmillan C (2013) Feminization laryngoplasty: assessment of surgical pitch elevation. Eur Arch Otorhinolaryngol 270:2695-2700 
11. Jacobson BH (1997) The voice handicap index (VHI): development and validation. Am J Speech Lang Pathol 6:66

12. Boersma P, Weenink D (2012). Praat: doing phonetics by computer [Computer program]. Version 5.3.15, retrieved 10 May 2012 from http://www.praat.org/

13. Carding PN, Wilson JA, MacKenzie K, Deary IJ (2009) Measuring voice outcomes: state of the science review. J Laryngol Otol 123:823-829

14. Matai V, Cheesman AD, Clarke PM (2003) Cricothyroid approximation and thyroid chondroplasty: a patient survey. Otolaryngol Head Neck Surg 128:841-847

15. Wagner I, Fugain C, Monneron-Girard L, Cordier B, Chabolle F (2003) Pitch-raising surgery in fourteen male-to-female transsexuals. Laryngoscope. 113:1157-1165

16. Remacle M, Matar N, Morsomme D, Veduyckt I, Lawson G (2011) Glottoplasty for male-to-female transsexualism: voice results. J Voice 25:120-123

17. Mastronikolis NS, Remacle M, Biagini M, Kiagiadaki D, Lawson G (2013) Wendler Glottoplasty: an effective pitch raising surgery in male-to-female transsexuals. J Voice 27:516-522
18. Anderson JA (2014) Pitch elevation in trangendered patients: anterior glottic web formation assisted by temporary injection augmentation. J Voice 28:816-821

19. Valo M (1994) Käsitykset ja Vaikutelmat Äänestä.Kuuntelijoiden Arviointia Radiopuheen Äänellisistä Ominaisuuksista. Thesis published in Finnish on Descriptions and Impressions of Voice. Evaluative Responses to Vocal Phenomena in Radio Speech. Studia Philologica Jyväskyäensia 33. Jyväskylä University, Jyväskylä

20. Leino T (1998) In search of 'optimal pitch': the lowest possible tone as the reference in the evaluation of speaking pitch in Finnish speakers. In: Proceedings 24th World Congr Int Assoc Logop Phoniatr, August 1998

21. Stoicheff ML (1981) Speaking fundamental frequency characteristics of nonsmoking female adults. J Speech Lang Hear Res 24:437-441 